\title{
Time coordination of standalone measurement instruments by synchronized triggering
}

\author{
Francesco Lamonaca, Domenico Luca Carnì, Domenico Grimaldi \\ Department of Computer Science, Modeling, Electronics and System Science, University of Calabria, Rende (CS), Italy
}

\section{ABSTRACT}

A Hardware Interface (HI) to synchronize the operations of standalone Measurement Instruments (MIs) in the absence of networking has been proposed in the recent literature. The synchronization accuracy achieved is one period of the clock equipping the HI. To improve the synchronization accuracy two solutions can be argued on the basis of the mathematical model of the delay between HIs. The first involves increasing the clock frequency; the second concerns the compensation of the phase delay between $\mathrm{HI}$ clocks. In this paper the second solution is adopted in order to: (i) reduce the energy consumption, and (ii) not increase the complexity of the hardware architecture. The phase delay compensation is obtained by introducing a programmable delay line after the $\mathrm{HI}$ clocks. The phase delay evaluation and the successive tuning of the delay line are performed in the synchronization phase of the HIs. Once synchronized, each $\mathrm{HI}$ is moved to the standalone $\mathrm{MI}$ to trigger it according to the common sense of time. During the execution of the measurement procedure, networking is not necessary. Experimental tests validate the correct operation of the upgraded $\mathrm{HI}$ architecture and indicate that the achievable synchronization accuracy is a low percentage of the HI clock period.

\section{Section: RESEARCH PAPER}

Keywords: Synchronization; Embedded hardware; Standalone Measurement Instrument; Distributed Measurement System

Citation: Francesco Lamonaca, Domenico Luca Carnì, Domenico Grimaldi, Time coordination of standalone measurement instruments by synchronized triggering, Acta IMEKO, vol. 4, no. 3, article 4, September 2015, identifier: IMEKO-ACTA-04 (2015)-03-04

Editor: Paolo Carbone, University of Perugia, Italy

Received February 16, 2015; In final form May 25, 2015; Published September 2015

Copyright: (C) 2015 IMEKO. This is an open-access article distributed under the terms of the Creative Commons Attribution 3.0 License, which permits unrestricted use, distribution, and reproduction in any medium, provided the original author and source are credited

Funding: This work was partially supported by the Italian Ministry of Economic Development by means of the RIDITT: "Italian Network for Innovation and Technology Transfer to Enterprises", under the project "DI.TR.IM.MIS Diffusione e trasferimento di tecnologie ad imprese nel settore delle misure".

Corresponding author: Domenico Luca Carnì, e-mail: dlcarni@dimes.unical.it

\section{INTRODUCTION}

In [1] the Hardware Interface (HI) architecture was proposed to bring the common sense of the time to the standalone Measurement Instruments (MIs) [2], [3] in operative scenarios in which no stable network is available. The time coordination of the operations of MIs is difficult when: (i) the network topology does not provide a continuous, reliable and stable link, wired or wireless, to the whole network [4]-[15], (ii) the installation of new wired or wireless links or repeaters would be expensive or not physically or technically possible [16]-[19], and (iii) a time constraint requires both fast and efficient solutions [20]-[22]. In these cases, no network services are available for the standalone MIs and synchronization services based on packet exchange as the std. IEEE1588 cannot be used [23].
The HI architecture includes a counter driven by a clock, and a Programmable Interface Controller (PIC). The HIs are synchronized by enabling the HI counters at the same time. After that, the HIs are moved and connected to the standalone MIs that can be in different places where no networking is available. The time coordination of the measurement operations is achieved by triggering the MIs in synchronized modality.

The software and hardware architecture of the HI are designed so that (i) the random effects of the operating system are avoided, and (ii) the polling cycle to check the trigger condition is reduced [24]-[26]. The result is that the synchronization accuracy between two triggers sent to the standalone MIs is one clock period.

In the paper, the mathematical model of the contribution of each hardware and software component to the synchronization delay is developed. The analysis of the mathematical model highlights that to improve the synchronization accuracy two 
solutions can be argued: (i) increasing the clock frequency, and (ii) reducing the phase delay between clocks.

The first solution involves problems in the design of the HI [27], and increases the power consumption [28].

The second solution is more advantageous than the first and it is developed in this paper. To this purpose, the hardware architecture of the $\mathrm{HI}$ is upgraded by adding the programmable delay line after the clock block. This delay line permits realignment of the clock signals of the HIs once their phase delays are previously evaluated.

The phase delay evaluation and the successive tuning of the delay line are performed in the synchronization phase of the HIs. The first step of the synchronization phase concerns the evaluation and compensation of the clock delays by tuning the programmable delay line. The second step concerns the synchronized start of the counter block, as in [1]. During the synchronization, communication between the HIs is required. During the triggering of the MIs communication among HIs is not required and the synchronized measurement procedure can be performed also in the absence of networking.

The paper is organized as follows: in Section 2 the hardware architecture and the performed logical operations of the upgraded HI are presented; in Section 3 the mathematical model of the synchronization delay is analysed; in Section 4 the experimental results are discussed; finally, conclusions are drawn in Section 5.

\section{OPERATIONS PERFORMED BY THE UPGRADED HI}

The upgraded HI compensates the delay between the trigger signals sent to the MIs, as in [1], as well as the clock phase delays, to further reduce the synchronization delay.

The upgraded HI is composed by five blocks (Figure 1):

(i) Clock Block: drives the Programmable Interface Controller (PIC) and the Counter Block;

(ii) programmable delay line: compensates the phase delay between two HI clocks;

(iii) 8-bit Counter block: feeds the PIC with synchronized impulses;

(iv) PIC: generates the synchronized trigger signal on the pin RB1 for the MI. Moreover, it tunes the programmable delay line in the synchronization phase on the basis of the information sent by the Master PC;

(v) board Rabbit RCM4400W: operates as Wi-Fi interface between the HI and the Master PC. It is used only for the initial synchronization, and after that is disconnected from the HI.

The synchronization between two HIs is executed by the Starter block (Figure 1) and the Master PC on the basis of the procedure described in Subsection 2.1.

\subsection{HI synchronization procedure}

The synchronization procedure is organized in two successive steps.

The first step requires the connection among the Master PC, two HIs and the Starter Block, as shown in Figure 1.

The Master PC commands: (i) each HI to pull down the pin TroK in order to inhibit the Start Count block, (ii) the Starter Block to measure the phase delay $\Delta t_{p h}$ between the clocks of $\mathrm{HI} \# 1$ and $\mathrm{HI} \# 2$ connected to the pins $\mathrm{Clk}_{1}$ and $\mathrm{Clk}_{2}$ of the Phase Delay Measurement Block (PDMB).

The PDMB is equipped by an internal counter with period $T_{\text {clk_starter }}=5 n s$ equal to $1 / 20$ of the $\mathrm{HI}$ clock period that is

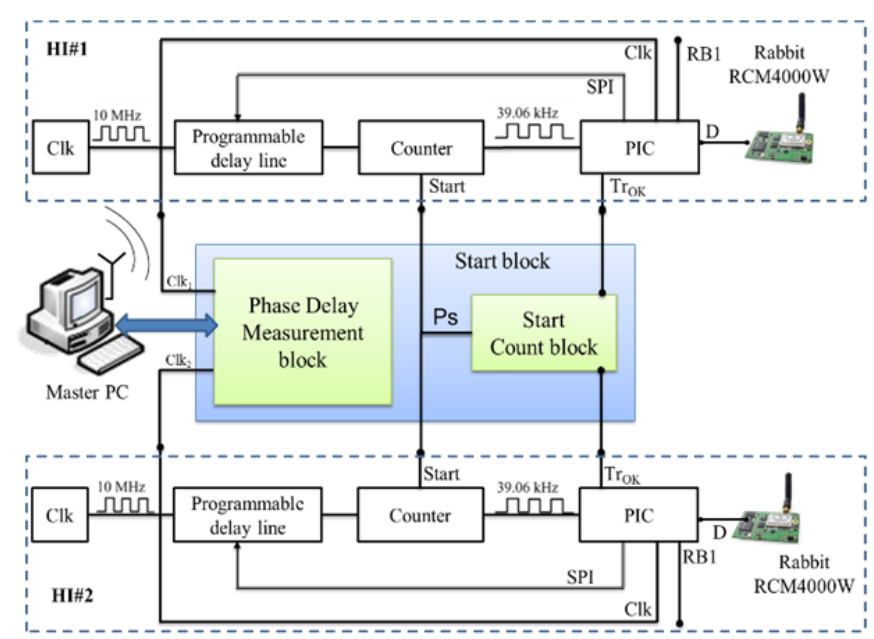

Figure 1. Block scheme of the connection among upgraded HIs, Starter Block, and Master PC in the synchronization phase.

100 ns. Therefore, the clock frequency of the starter block is $200 \mathrm{MHz}$. The mean value $\overline{\Delta t}_{p h}$ of 100 measures of the clock phase delay is sent to the Master PC by the Universal Serial Bus connection, and, successively, by the Wi-Fi interface to the HIs for tuning the programmable delay line.

Experimental tests indicate that after the synchronization, due to the hardware and software architecture of the $\mathrm{HI}$ and the starter block, the phase delay is lower than the resolution of the starter block (5 ns). In particular, by using a Digital Storage Oscilloscope with resolution $0.1 \mathrm{~ns}$, the measured clock phase delay is $2.2 \mathrm{~ns}$ with standard deviation $0.9 \mathrm{~ns}$. Increasing the number of measurements does not provide any advantage because the mean value does not significantly change and the standard deviation is less than the resolution of the starter block. The tuning procedure ends if:

$\overline{\Delta t}_{p h} \leq \max \left(T_{P D L}, T_{c l k_{\text {_starter }}}\right)$

where $T_{P D L}$ is the resolution of the programmable delay line. This condition ensures that the tuning procedure ends in the case the measured phase delay cannot be further evaluated, if $\overline{\Delta t}_{p h} \leq T_{c l k_{\text {_starter }}}$, or compensated, if $\overline{\Delta t}_{p h} \leq T_{P D L}$.

Once the phase delay is compensated, the second step of the synchronization procedure starts. As in [1], the Master PC sends the following trigger conditions to the two HIs:

- $n_{i p p}$ number of impulses that the PIC must count before triggering the $\mathrm{MI}$,

- $n_{m}$ number of repetitive measures to be executed by the MI,

- $v_{m}$ number of impulses between two consecutive measures.

In order to execute synchronized measurement operations it is necessary that $n_{i p}$ and $n_{m}$ are identical for both the HIs. Once this information is received, the HI pulls up the pin $\operatorname{Tr}_{\mathrm{OK}}$.

When both the pin $\mathrm{Tr}_{\mathrm{OK}}$ of $\mathrm{HI} \# 1$ and $\mathrm{HI} \# 2$ are high, the pin Ps of the Start Count block is pulled up and the Counter block of each HI starts.

The signal on pin Ps is sent to the HIs in parallel connection, guaranteeing that the delay between the start of the Counter blocks is determined only by their hardware characteristics.

Once the PICs receive the impulse, the HIs can be disconnected from the Starter block and connected to the standalone MIs. 
The Wi-Fi connection is involved in the synchronization process of the HI only for the transmission of the trigger generation parameters $\left(n_{i t p}, n_{m}, v_{m}\right)$ and the measured phase delay for the tuning of the programmable delay line. Therefore, the Wi-Fi interface does not influence the synchronization accuracy. As a consequence, any boards implementing the conversion Wi-Fi-Serial communication can be used.

\subsection{Triggering procedure}

The time coordination among the operations executed by the MIs is obtained by the synchronized trigger signals sent by the HIs.

At the beginning, each HI set $n_{i t}$ and after that generates the trigger signal. Successively, all the HIs start the count simultaneously. The program set up in the PIC counts the counter impulses and checks whether the actual count is equal to the set-up value. These operations are performed before the successive impulse occurs. This reduces the effect of the polling to check the trigger condition.

Figure 2 shows the block diagram of the operation performed by the PIC:

1) the internal variable $i$ is incremented;

2) if $i=n_{i \phi p}$, then $\mathrm{HI}$ sends the trigger signal by generating an impulse signal in the RB1 line, and the new values are updated $n_{i p}=n_{i \phi p}+v_{m}, n_{m}=n_{m}-1$;

3) else go to step 1 ;

4) if $n_{m}=0$, the triggering procedure ends.

\section{SYNCHRONIZATION DELAY ANALYSIS}

The synchronization delay $\Delta T$ between two triggers is determined by the hardware architecture of the two HIs and the time needed to execute their operation (Figure 3). It is [1]:

$$
\begin{aligned}
\Delta T & =\left(n_{c} T_{c 1}+n_{p} T_{p 1}+E_{P 1}\right)+ \\
& -\left(n_{c} T_{c 2}+n_{p} T_{p 2}+E_{P 2}\right)+O_{C}+O_{P} ;
\end{aligned}
$$

where:

- $T_{c 1}$ and $T_{c 2}$ are the clock periods of HI\#1 and HI\#2, respectively;

- $\quad T_{p 1}$ and $T_{p 2}$ are the clock periods of PICs equipping HI\#1 and $\mathrm{HI} \# 2$, respectively;

- $\quad n_{c}$ is the counting number of HI\#1 and HI\#2, equal to

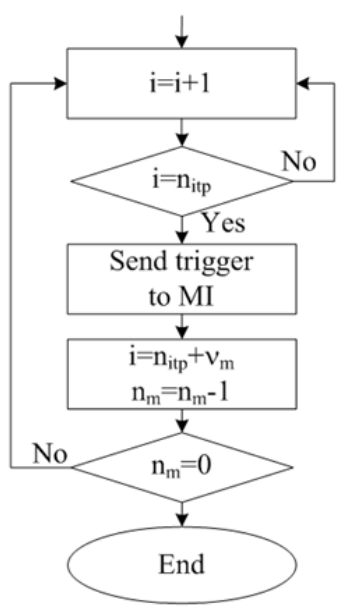

Figure 2. Block diagram of the performed operation by each $\mathrm{HI}$ for the synchronized generation of the trigger signal.

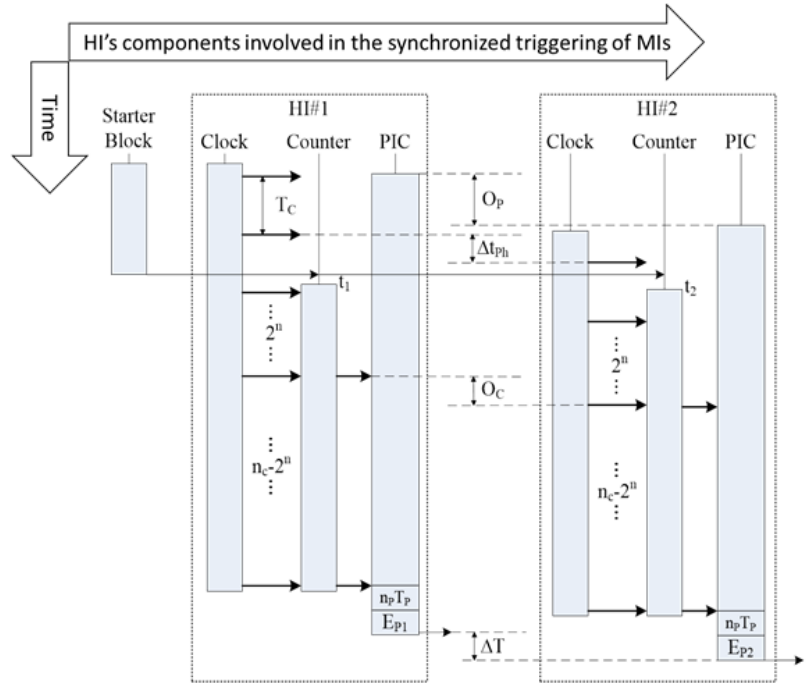

Figure 3. Unified modelling language diagram of the operations executed by the $\mathrm{HI}$ components involved in the synchronized triggering of the Mls.

$n_{i t p} 2^{n}$ where $n$ is the number of bit of the counter;

- $n_{p}$ is the number of elementary operations to execute the triggering procedure in the PIC;

- $O_{C}$ is the start offset time between the counting on HI\#1 and HI\#2. It depends on $\Delta t_{P h}$ and the time instant $t_{1}$ and $t_{2}$ (Figure 4) corresponding to the opening of the counter
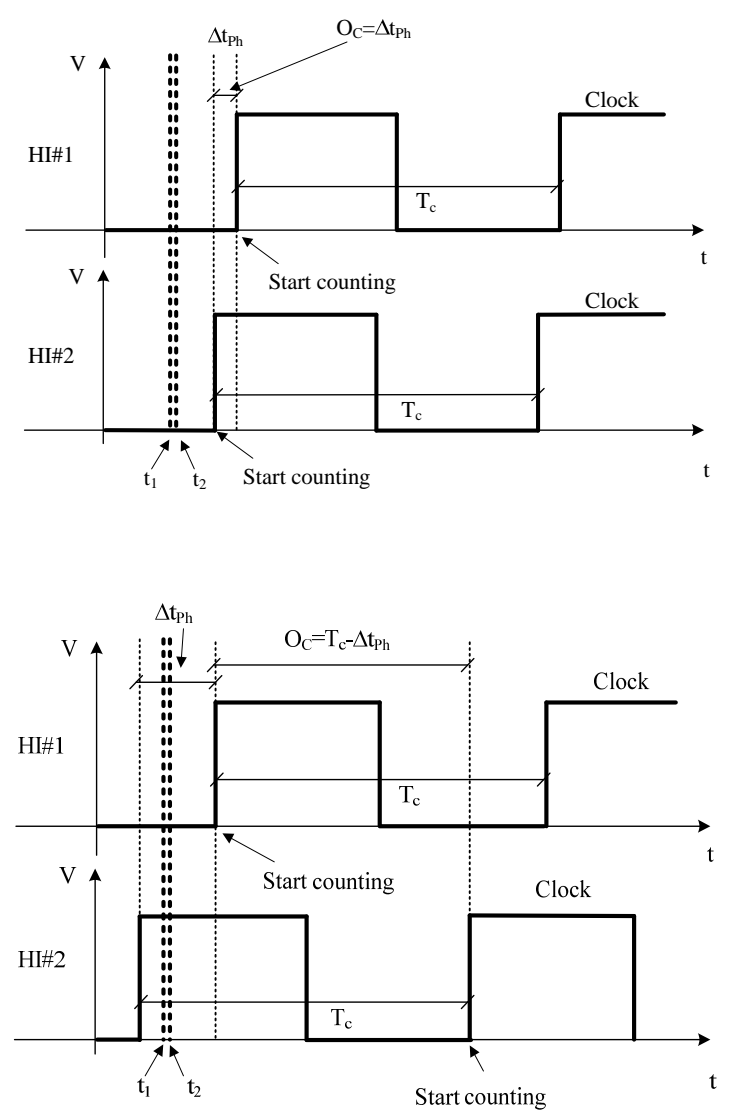

Figure 4. Delay between the opening of the counter gate and the start of counting. a) $t_{1}$ and $t_{2}$ occur before the raising of the two clock signals, b) $t_{1}$ and $t$, occur between the raising of the two clock signals. 
gate of $\mathrm{HI} \# 1$ and $\mathrm{HI} \# 2$, respectively;

- $O_{P}$ is the start offset time between two PICs;

- $E_{P 1}$ and $E_{P 2}$ are the time intervals required by the hardware of the two PICs to pull up the pin RB1 and, then, trigger the MI.

Owing to the synchronized start of the counting, $O_{P}$ has no influence in the evaluation of $\Delta T$.

By considering the vector $\bar{x}$ made up of: $n_{c}, n_{p}, T_{c 1}, T_{c 2}, T_{p 1}$, $T_{p 2}, E_{P 1}, E_{P 2}, O_{C}$, the variation of the synchronization time delay (1) evaluated according to [29] is:

$$
\begin{aligned}
\Delta_{\Delta T}= & \frac{\sqrt{\left(\left.\left(n_{c}\right)\right|_{\bar{x}} \Delta_{T_{c 1}}\right)^{2}+\left(\left.\left(n_{c}\right)\right|_{\bar{x}} \Delta_{T_{C 2}}\right)^{2}+\left(\left.\left(n_{p}\right)\right|_{\bar{x}} \Delta_{T_{p 1}}\right)^{2}+}}{+\left(\left.\left(-n_{p}\right)\right|_{\bar{x}} \Delta_{T_{P 2}}\right)^{2}+\Delta_{O_{C}}^{2}+\Delta_{E_{P 1}}^{2}+\Delta_{E_{P 2}}^{2}}
\end{aligned}
$$

where:

- $\Delta_{T c 1}$ and $\Delta_{T c 2}$ are the variation range of the values of $T_{c l}$ and $T_{c 2}$, respectively,

- $\Delta_{T p 1}$ and $\Delta_{T p 2}$ are the variation range of the values of $T_{p 1}$ and $T_{p 2}$, respectively,

- $\Delta_{E^{P_{1}}}$ and $\Delta_{E^{P_{2}}}$ are the variation range of the values of $E_{P 1}$ and $E_{P 2}$, respectively.

- $\Delta_{O c}$ is the variation range of the values of $O_{C}$.

Since the two HIs have the same hardware, it is:

$T_{C 1}=T_{C 2}=T_{C}, \quad T_{P 1}=T_{P 2}=T_{P}$

$\Delta_{T_{C 1}}=\Delta_{T_{C 2}}=\Delta_{T_{C}}, \quad \Delta_{T_{P 1}}=\Delta_{T_{P 2}}=\Delta_{T_{P}}$

Moreover, absence of conditional jumps and correlation between parameters can be assumed. In fact, to check the trigger condition in the software set up in the PIC there are no conditional jumps and the parameters in (2) are related to independent devices.

The variation range of $\Delta_{T c}$ and $\Delta_{T p}$ is according to the propagation delay of the hardware components and can be evaluated by their datasheet.

The variation range $\Delta_{O c}$ depends on $\Delta t_{P b}$. In particular two cases can occur:

- if $t_{1}$ and $t_{2}$ are before the raising edge of the clock signals (Figure 4a), $O c=\Delta t_{P b}$. Indeed, the counters of HI\#1 and HI\#2 are sensitive to the raising edges of the clocks that have lower delay;

- otherwise $O c=T c-\Delta t_{P b}$ (Figure $\left.4 \mathrm{~b}\right)$. The gate of the HI\#1 counter is opened before the occurrence of the raising edge of the HI\#1 clock, the gate of HI\#2 counter is opened after the occurrence of the raising edge of the HI\#2 clock. Consequently, the HI\#2 counter will be sensitive to the successive raising edge of the HI\#2 clock.

Since $\Delta t_{P b}$ varies in the range $[0, T c], \Delta_{O c}$ varies in the range [0, Tc], also. Decreasing $\Delta t_{P h}$, the probability that $O c=\Delta t_{P h}$ increases (Figure $4 \mathrm{a}$ ). As a consequence, $O_{C}$ value is decreased.

The programmable delay line permits the reduction of the variation range of $\Delta t_{P h}$ according to its resolution. As a consequence of the parallel connection among Starter Block and the two HIs, $\left|t_{2}-t_{1}\right|$ is of some ps and then negligible with respect to $\Delta t_{P b}$. In fact, $\left|t_{2}-t_{1}\right|$ is determined by the propagation delay in the two cable lengths of $(0.150 \pm 0.001) \mathrm{m}$ connecting the pin Ps of the Starter Block with the start input pin of the HIs, and the difference between times to change the gate states. Owing to the propagation speed of the signal, the difference between $t_{1}$ and $t_{2}$ is about 5 ps. The difference between the times to change the gate states is in the order of 10 ps. Therefore, the difference between $t 1$ and $t 2$ is almost $15 \mathrm{ps}$, negligible with respect to $T c=100 \mathrm{~ns}$. It can be assumed that $t_{1} \approx t_{2}=t^{*}$. Due to periodicity of the clock signal the probability that $t^{*}$ is included in the time interval $T_{C}$ is 1 . The corresponding probability density function is:

$$
f(t)=\frac{1}{T_{C}} .
$$

Consequently, the probability $\mathbf{p}$ that $t^{*}$ occurs in the time interval $\Delta t_{P b}$ is [30]:

$\mathbf{p}=P\left(0<t^{*}<\Delta t_{P h}\right)=\int_{0}^{\Delta t_{p h}} f(t) d t=\frac{\Delta t_{P h}}{T_{C}}$.

By taking into consideration that at the end of the synchronization procedure $\Delta t_{P b}<5 \mathrm{~ns}$ and $T_{c}=100 \mathrm{~ns}$, it results in $\mathbf{p}<5 \%$.

Therefore, by using the programmable delay line, both $O_{C}$ and $\Delta_{O c}$ can be reduced to the order of ns.

\section{EXPERIMENTAL TESTS}

Experimental tests are developed in order to validate the correctness of the operations performed by the upgraded HI and to evaluate the time delay between two trigger signals.

The HI is equipped with a thermal compensated clock with period $100 \mathrm{~ns}$.

The measurement of the time delay between the trigger signals is executed by connecting the pins RB1 of the HI\#1 and

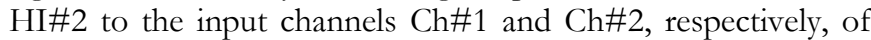
the Digital Storage Oscilloscope (DSO) Tektronix TDS7154B, as shown in Figure 5.

The sampling frequency of the DSO is set to $10 \mathrm{GS} / \mathrm{s}$, to ensure a sampling period equal to $0.1 \mathrm{~ns}$.

The Master PC is connected by a GPIB interface bus to the DSO, and sends the trigger conditions to the two HIs by Wi-Fi connection. In the experimental tests the trigger conditions are the same for both HIs.

The time delay between the triggers is evaluated by means of the number of samples occurring between the crossing of the established threshold by the two input signals. In particular, denoting $i$ and $j$ the indexes corresponding to the samples crossing the threshold, and $d t$ the sampling period of DSO, the time delay is:

$$
\Delta t=|(i-j) d t| \text {. }
$$

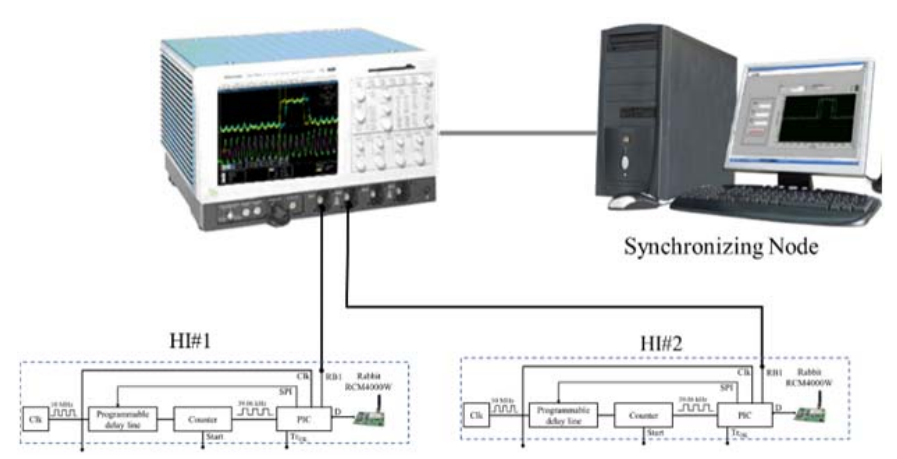

Figure 5. Experimental setup to evaluate the delay between the trigger signals generated by two HIs. 
In the first set of experiments the following parameters are set: $n_{i p}=351562, n_{m}=4000$, and $v_{m}=39062$. With this setup, the first trigger is generated after $n_{i t p} * 2 * 100 \mathrm{~ns}=9 \mathrm{~s}$ from the end of the HIs synchronization. This time is enough to disconnect the HIs from the Starter Block and to connect them to the MIs. The successive 4000 triggers are generated each $v_{m}{ }^{*}$ $28 * 100 \mathrm{~ns}=1 \mathrm{~s}$.

Figure 6a) shows the trend of $\Delta T$ versus the acquisition time, Figure $6 \mathrm{~b}$ ) its histogram in the case the synchronization of the two HIs is performed at the beginning and the programmable delay line is not used. In this case $\Delta T$ is characterized by the mean value $\mu=62.3$ ns and standard deviation $\sigma=0.4$ ns. Similarly, Figures $6 \mathrm{c}$ ) and $6 \mathrm{~d}$ ) show the trend of $\Delta T$ and its histogram evaluated by repeating the synchronization procedure. In this case $\Delta T$ is characterized by $\mu=86.1$ ns and $\sigma=0.4$ ns.

Figure 6 shows that, without the programmable delay line, the mean value of the delay between the $\mathrm{HI}$ triggers depends on the synchronization procedure and does not change during the observation time. In the two different experiments the widths of the variation ranges are comparable.

Figure 7a) shows the trend versus the acquisition time of $\Delta T$, Figure $7 \mathrm{~b}$ ) its histogram in the case the synchronization of the two HIs is performed at the beginning and the programmable delay line is used. In this case $\Delta T$ is characterized by $\mu=5.8$ ns and $\sigma=0.4$ ns. Similarly, Figures 7c) and d) show the trend of $\Delta T$ and its histogram evaluated by repeating the synchronization procedure. In this case $\Delta T$ is characterized by $\mu=7.3$ ns and $\sigma=0.4$ ns.

These results confirm the effectiveness of the proposed synchronization procedure to compensate for $\Delta t_{p b}$.

The results of repeated experiments highlight: (i) the proper operation of the upgraded starter block makes that the delayed start of the PIC does not influence the synchronization accuracy and (ii) with the upgraded $\mathrm{HI}$ architecture, the delay between the trigger signals is a low percentage of the clock period of HI.

\section{CONCLUSIONS}

The synchronization accuracy of the Hardware Interfaces (HIs) bringing the common sense of the time to standalone MIs [1] is improved.

The HI architecture is upgraded by adding the programmable delay line after the clock in order to reduce the phase delay that is the main source degrading the synchronization accuracy.

The synchronization procedure is also upgraded to provide the measurement of the phase delay that must be compensated by adjusting the delay line.

The experimental results indicate that, by considering the actual realization of the upgraded HI, the accuracy obtained is a low percentage of the HI clock period, whereas with the previous configuration it is one clock period.

The on-going activity is devoted to developing a $\mathrm{HI}$ able to work in scenarios with or without networking. When the network is not available, the HI works as described in the paper. When the network is available, the HI will implement a synchronization procedure based on packet exchange.
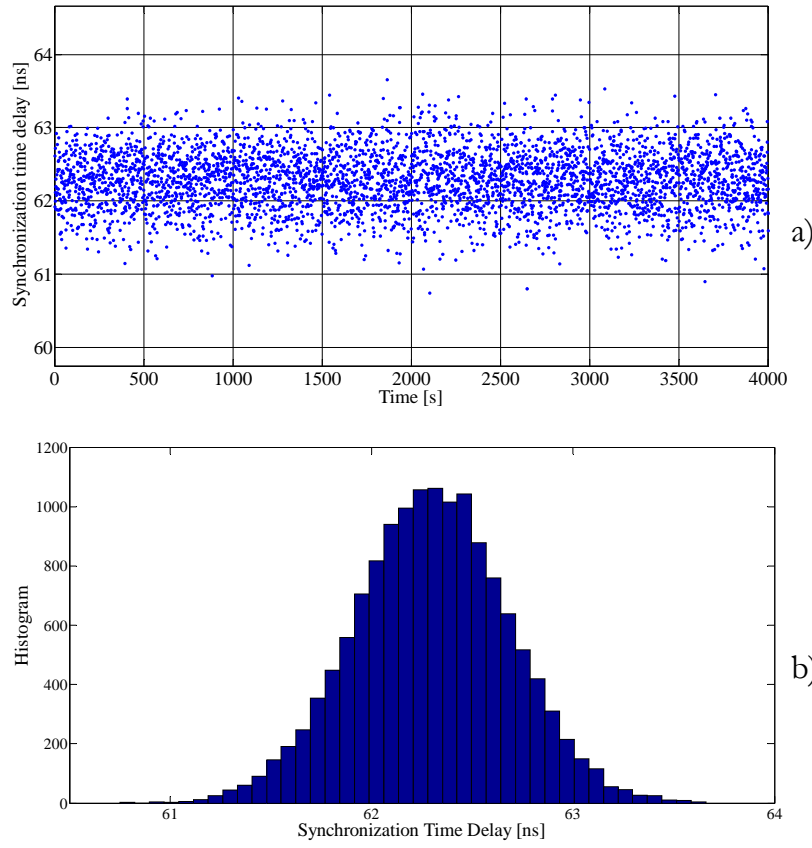

b)
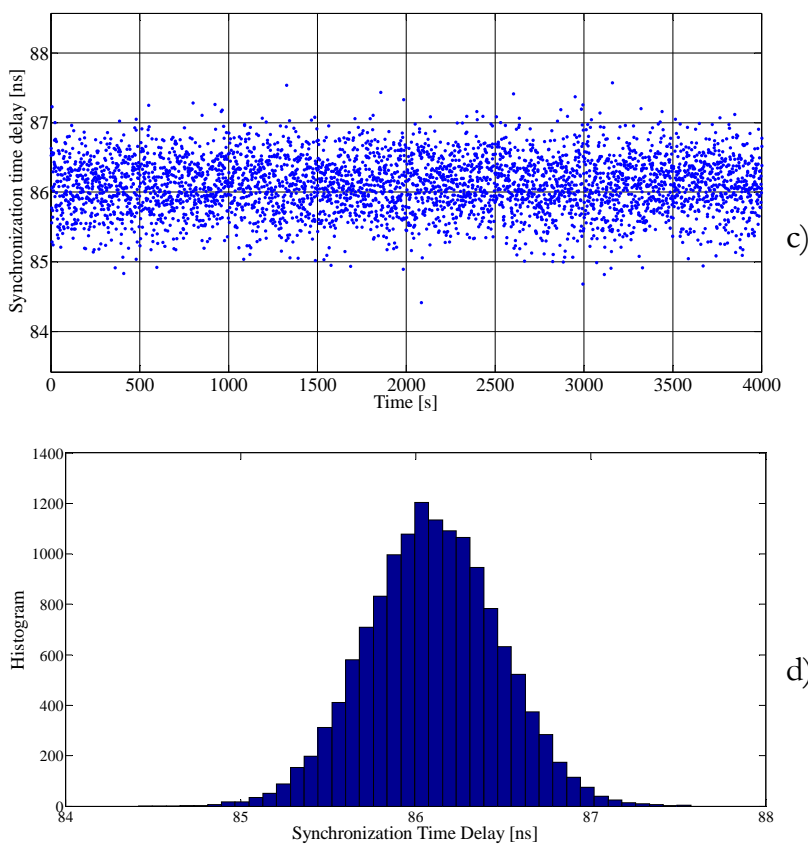

Figure 6. Trends of $\Delta T$ versus the acquisition time a), and their histogram b), in the case where the synchronization of the two Hls is performed at the beginning and the programmable delay line is not used. c) and d) are evaluated by repeating the synchronization procedure.

\section{ACKNOWLEDGEMENT}

This work was partially supported by the Italian Ministry of Economic Development by means of the RIDITT: "Italian Network for Innovation and Technology Transfer to Enterprises", under the project "DI.TR.IM.MIS Diffusione e trasferimento di tecnologie ad imprese nel settore delle misure".

\section{REFERENCES}

[1] F. Lamonaca, D. L. Carnì, D. Grimaldi, "New hardware interface to synchronize stand-alone measurement instruments," Proc. of: 20th IMEKO TC4 International Symposium and 18th International Workshop on ADC Modelling and Testing 


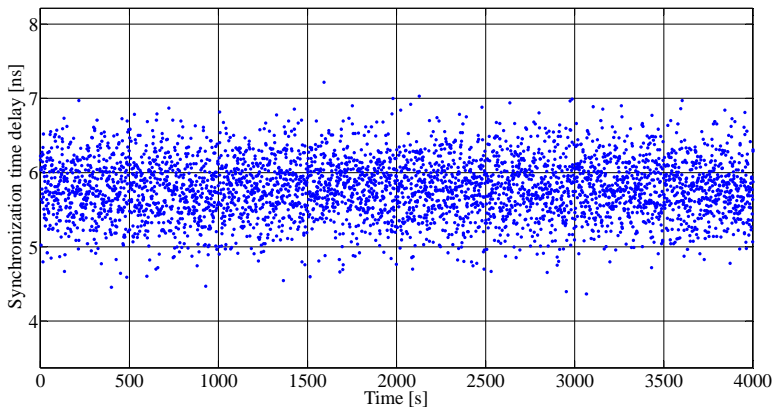

a)

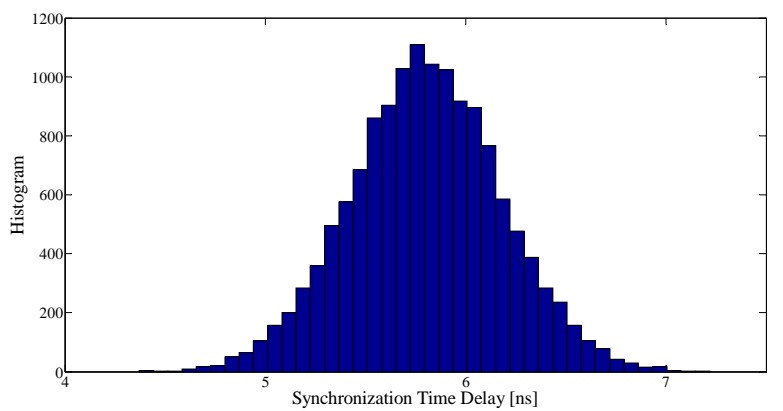

b)
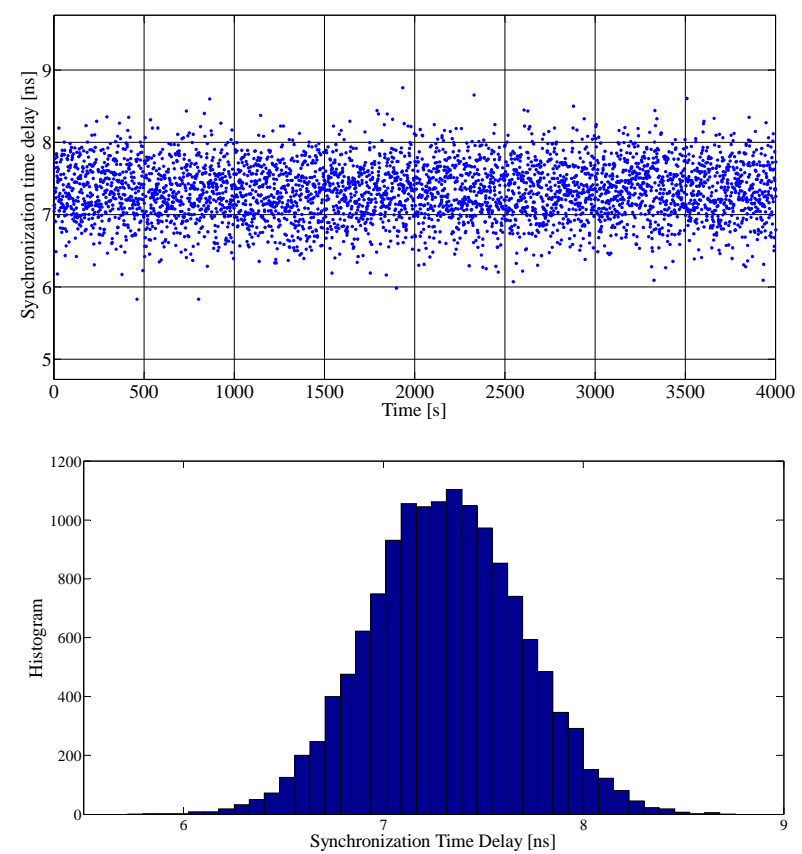

Figure 7. Trends of $\Delta T$ versus the acquisition time a), and their histogram b), in the case where the synchronization of the two HIs is performed at the beginning and the programmable delay line is used. c) and d) are evaluated by repeating the synchronization procedure.

Research on Electric and Electronic Measurement for the Economic Upturn, Benevento, Italy, 2014.

[2] D. L. Carnì, D. Grimaldi, G. Guglielmelli, F. Lamonaca, "Synchronization of Measurement Instruments co-Operating into the W-DMS," Proc. of: IEEE Instrumentation and Measurement Technologies Conference, Warsaw, Poland, 2007.

[3] D. Grimaldi , M. Marinov, "Distributed measurement systems", Measurement, vol. 30, pp. 279-287, 2001.

[4] G. Shuo, H. Liang, G. Yu, J. Bo, H. Tian, "Opportunistic Flooding in Low-Duty-Cycle Wireless Sensor Networks with Unreliable Links", IEEE Transactions on Computers, vol. 63, pp. 2787-2802, 2014.

[5] Z. Jun, "Minimum node degree and k-connectivity in wireless networks with unreliable links", in Proc. of IEEE International Symposium on Information Theory (ISIT), 2014, pp. 246-250.
[6] Z. Shizhen, W. Xinbing, "Node Density and Delay in LargeScale Wireless Networks With Unreliable Links", IEEE/ACM Transactions on Networking, vol. 22, pp. 1150-1163, 2014.

[7] I. H. Hou, "Broadcasting Delay-Constrained Traffic Over Unreliable Wireless Links With Network Coding", IEEE/ACM Transactions on Networking, 2014.

[8] F. De Rango, A. Panzarella, S. Marano, "Controlled Interference Mitigation MAC for UWB Networks with Quality of Service Support", in Proc. of IEEE International Conference on Mobile Adhoc and Sensor Systems. MASS 2007. , 2007, pp. 1-3.

[9] F. Rango, A. Socievole, S. Marano, "Exploiting online and offline activity-based metrics for opportunistic forwarding", Wireless Networks, pp. 1-17, November 82014.

[10] S. El Khediri, N. Nasr, A. Kachouri, A. Wei, "Synchronization in wireless sensors networks using balanced clusters", in Proc. of 6th Joint IFIP Wireless and Mobile Networking Conference (WMNC) 2013, pp. 1-4.

[11] L. Kebin, M. Qiang, L. Haoxiang, C. Zhichao, L. Yunhao, "Endto-End Delay Measurement in Wireless Sensor Networks without Synchronization", in Proc. of IEEE 10th International Conference on Mobile Ad-Hoc and Sensor Systems (MASS), 2013, pp. 583-591.

[12] P. Kieu-Ha, T. Huy, J. Tiete, L. Tran, K. Steenhaut, "Lowoverhead time synchronization for schedule-based multi-channel wireless sensor networks", in Proc. of 19th IEEE Workshop on Local \& Metropolitan Area Networks (LANMAN), , 2013, pp. 1-6.

[13] J.-t. Guo, M.-y. Hou, Y.-x. Wang, G.-f. Zhu, G.-b. Zou, C.-h. Zhai, X.-h. Liu, N. Zhang, "Application of IEEE1588 in time synchronizing system of smart distribution grid", in Proc. of 12th IET International Conference on Developments in Power System Protection (DPSP 2014), 2014, pp. 1-4.

[14] D. Kashyap , T. K. Jain, "Comparative analysis of time synchronization schemes for wireless sensor networks", in Proc. of 2014 International Conference on Signal Propagation and Computer Technology (ICSPCT), 2014, pp. 106-109.

[15] A. Puhm, M. Kramer, P. Moosbrugger, M. Horauer, "Problems and solutions for refitting a sensor network with IEEE1588 clock synchronisation", in Proc. of IEEE Emerging Technology and Factory Automation (ETFA), 2014, pp. 1-7.

[16] F. Lamonaca, G. Pizzuti, A. Arcuri, "Environmental Parameters in the National Gallery of Cosenza: A Case Of Study," Proc. of: 4th Imeko TC19 Symposium on Environmental Instrumentation and Measurements, Lecce, Italy, 2013.

[17] M. L. Vincent, F. Kuester, T. E. Levy, "OpenDig: In-field data recording for archaeology and cultural heritage", in Proc. of Digital Heritage International Congress (DigitalHeritage), 2013, pp. 539542.

[18] D. L. Carnì, D. Grimaldi, F. Lamonaca, "Improved synchronizing procedure of PDAS to delivery the common sense of the time to stand alone measurement instrument", in Proc. of 19th IMEKO World Congress 2009, 2009, pp. 1335-1340.

[19] F. Lamonaca, G. Pizzuti, N. Arcuri, A. M. Palermo, R. Morello, "Monitoring of environmental parameters and pollution by fungal spores in the National Gallery of Cosenza: A case of study", Measurement, vol. 47, pp. 1001-1007, 2014.

[20] C. H. Cuadra, N. Shimoi, T. Nishida, M. Saijo, "Estimation of dynamic properties of traditional wooden structures using new bolt sensor", in Proc. of 13th International Conference on Control, Automation and Systems (ICCAS), 2013, pp. 1593-1598.

[21] L. Gang, S. J. Qin, Z. Donghua, "A New Method of Dynamic Latent-Variable Modeling for Process Monitoring", IEEE Transactions on Industrial Electronics, vol. 61, pp. 6438-6445, 2014.

[22] M. Lydon, S. E. Taylor, D. Robinson, P. Callender, C. Doherty, S. K. T. Grattan, E. J. O'Brien, "Development of a Bridge Weigh-in-Motion Sensor: Performance Comparison Using Fiber Optic and Electric Resistance Strain Sensor Systems", IEEE Sensors Journal, vol. 14, pp. 4284-4296, 2014.

[23] "IEEE Standard for a Precision Clock Synchronization Protocol for Networked Measurement and Control Systems", IEEE Std 1588-2008 (Revision of IEEE Std 1588-2002), pp. c1-269, 2008. 
[24] F. Lamonaca, D. Grimaldi, "Trigger Realignment by Networking Synchronized Embedded Hardware", IEEE Transaction on Instrumentation and Measurement, vol. 62, pp. 38-49, 2013.

[25] F. Lamonaca, A. Gasparri, E. Garone, D. Grimaldi, "Clock Synchronization in Wireless Sensor Network With Selective Convergence Rate for Event Driven Measurement Applications", IEEE Transactions on Instrumentation and Measurement, vol. 63, pp. 2279-2287, 2014.

[26] F. Lamonaca, D. Grimaldi, R. Morello, "Dynamic Scheduling of Trigger Command for sub- $\mu$ s alignment Accuracy in Distributed Measurement System", IEEE Transaction on Instrumentation and Measurement, vol. 63, pp. 1795 - 1803, 2014.
[27] Electromagnetic Compatibility of Integrated Circuits: Springer Science+Business Media, 2006.

[28] A. Y. Feinstein, M. A. Thornton, F. Kocan, "System-on-chip power consumption refinement and analysis", in Proc. of 2007 IEEE Dallas/CAS Workshop on System-on-Chip (SoC): Design, Applications, Integration, and Software, DCAS-07, 2007, pp. 81-84.

[29] I. O. f. Standardization, Guide to the Expression of Uncertainty in Measurement (GUM), 1998.

[30] M. H. DeGroot, M. J. Schervish, X. Fang, L. Lu, D. Li, Probability and statistics vol. 2: Addison-Wesley Reading, MA, 1986. 